\title{
Subspace-based identification of a distributed nonlinearity in time and frequency domains
}

\author{
D. Anastasio ${ }^{1}$, S. Marchesiello ${ }^{1}$, J.P. Noël ${ }^{2}$, G. Kerschen ${ }^{2}$ \\ ${ }^{I}$ Department of Mechanical and Aerospace Engineering, Politecnico di Torino, Corso Duca degli Abruzzi 24, 10129 Torino, Italy. \\ ${ }^{2}$ Space Structures and Systems Laboratory, Department of Aerospace and Mechanical Engineering, University of Liège, Chemin des \\ Chevreuils 1, 4000 Liège, Belgium.
}

\begin{abstract}
Nonlinear system identification has become of great interest during the last decades. However, a common and shared framework is not present yet, and the identification may be challenging, especially when real engineering structures are considered with strong nonlinearities. Subspace methods have proved to be effective when dealing with local nonlinearities, both in time domain (TNSI method) and in frequency domain (FNSI method). This study reports an improvement for both methods, as a first attempt to account for distributed nonlinearities, which is still an open question in the research community. A numerical beam under moderately large oscillations that exhibits geometric nonlinearity is considered. The object of the identification process is to exploit its behavior through the correct identification of the parameters that define the nonlinearity. Results show a high level of confidence between the two methods, and suggest that a more complete analysis of distributed nonlinear phenomena can be conducted based on these approaches.
\end{abstract}

Keywords: nonlinear system identification, subspace identification, distributed nonlinearity, geometric nonlinearity, nonlinear beam.

\section{Introduction}

Identification of nonlinear dynamical systems has been thoroughly investigated in recent years. The number of methods developed is large and, generally, no method exists to be adopted for any type of nonlinearity, system complexity or exciting force [1]. However, a basic distinction on the nature of the nonlinearity can be done between local nonlinearities (clearances, loosing connections, ...) and distributed nonlinearities. A few examples of distributed nonlinearities are material (or constitutive), inertia, geometric [2]. In particular, the geometric nonlinearity is associated with large deformations in solids, resulting in nonlinear strain-displacement relations. This case has become frequent during the last years, due to a continual interest to expand the performance envelope of structures, which brings the need for designing lighter, more flexible, and consequently, more nonlinear structural elements [3, 4]. In this framework, system identification plays a key role as a tool to gather information about the dynamics of the system.

This study refers to a simple nonlinear beam under large transversal oscillations, which bring geometric nonlinearity. This should be considered as a first attempt to identify distributed nonlinearities, which is still an open point in the research community. Two methods are used, both based on subspace identification [5]: TNSI (Time Nonlinear Subspace Identification) [1,6] and FNSI (Frequency Nonlinear Subspace Identification) [7-8]. The main difference between TNSI and FNSI is the domain in which they work, which is time for the first and frequency for the second. They both proved to be effective in the identification of local nonlinearities [9], thus an extension to a case of distributed nonlinearity is here suggested. 


\section{Beam under large lateral oscillations}

A 2D Euler-Bernoulli beam with large lateral deflection is considered. The governing equation is [2]:

$$
\mu \frac{\partial^{2} w}{\partial t^{2}}+E I \frac{\partial^{4} w}{\partial x^{4}}-\frac{E A}{2 L}\left(\int_{0}^{L}\left(\frac{\partial w}{\partial x}\right)^{2} d x\right) \frac{\partial^{2} w}{\partial x^{2}}=f(t) \delta\left(x-x_{F}\right)
$$

Where $\mu$ is the linear mass, $E$ is the Young's Modulus, $I$ is the moment of intertia, $A$ is the section, $L$ is the length, $x_{F}$ is the forcing position and $w$ is the lateral displacement. The third term in eq. (1) expresses the nonlinearity, and can be seen as a variable axial load generated by the lateral movement. The solution to eq. (1) can be found applying the modal transformation expressed in eq. (2).

$$
w(x, t)=\sum_{j=1}^{\infty} \phi_{j}(x) \eta_{j}(t), \quad \phi_{j}=\mathrm{j}^{\text {th }} \text { eigenmode, } \quad \eta_{j}=\mathrm{j}^{\text {th }} \text { modal coordinate }
$$

The mode shapes $\phi_{j}$ depend on the boundary conditions at the ends of the beam, and analytic expression are available for the most common ones. A set of $\mathrm{N}$ equations is obtained substituting eq. (2) in eq. (1) and considering $\mathrm{N}$ modes, as expressed in eq. (3)

$$
m_{j} \ddot{\eta}_{j}+k_{j} \eta_{j}-\frac{E A}{2 L} \sum_{r=1}^{N}\left(c_{1, r}^{n l} \eta_{r}^{2}\right) c_{2, j}^{n l} \eta_{j}=Q_{j} \quad j=1, \ldots, N
$$

Where $Q_{j}=\phi_{j}^{\mathrm{T}} f$ are the modal forces, $m_{j}$ is the modal mass, $k_{j}$ is the modal stiffness and $c_{1, r}^{n l}, c_{2, j}^{n l}$ are the nonlinear coefficients. These depend on the boundary conditions, and can be generally expressed as:

$$
c_{1, r}^{n l}=\int_{0}^{L} \phi_{r}^{\prime}(x) d x, \quad c_{2, j}^{n l}=\int_{0}^{L} \phi_{j}(x) \phi_{j}^{\prime \prime}(x) d x
$$

These coefficients are the objective of the identification process implemented in the following example.

\section{Nonlinear System Identification}

TNSI and FNSI are applied to a numerical clamped-clamped beam under large lateral oscillations, whose characteristics are reported in Table 1. The methodology here explained can be applied also considering other boundary conditions which verify the hypothesis of self-adjoint operators for mass, stiffness and damping. The goal of the identification process is to extract the modal parameters of the underlying linear system and the coefficients that express the nonlinearity.

Table 1: Characteristics of the beam

\begin{tabular}{lll}
\multicolumn{3}{c}{ Table 1: Characteristics of the beam } \\
\hline Length $(\mathrm{mm})$ & $L$ & 300 \\
Section $\left(\mathrm{mm}^{2}\right)$ & $b \cdot h$ & $30 \cdot 1$ \\
Young's Modulus $(\mathrm{GPa})$ & $E$ & 200 \\
\hline
\end{tabular}

The numerical test is performed applying a periodic random excitation to the beam and measuring the response in 10 points by numerical integration. Also, a reasonable amount of Gaussian noise is added to the response. The identification is performed on the modal equations, thus a first low-excitation test is performed to extract the linear mode shapes of the beam, called $\phi^{\exp }$. It should be noticed that an analytical expression of the eigenmodes is not needed, assuming that a linear test can be performed on the structure. The experimental modal coordinates can be expressed as:

$$
\boldsymbol{\eta}^{\exp }=\operatorname{pinv}\left(\boldsymbol{\phi}^{\exp }\right) \boldsymbol{w}^{\exp }
$$

Once the modal coordinates are extracted, the identification can be performed starting from eq. (3) and remembering that both TNSI and FNSI treat the nonlinear force as a feedback. One of the advantages of the presented methodology is that the set of $\mathrm{N}$ equations are linearly independent, which means that the identification can be performed on each mode separately. This speeds up the calculations, as avoids the computation of huge matrices due to high model orders. An exemplary result of the identification considering the first 3 modes is depicted in Fig. 1. Both the FRF and the nonlinear coefficients are well identified, which proves the goodness of the adopted methodology. 

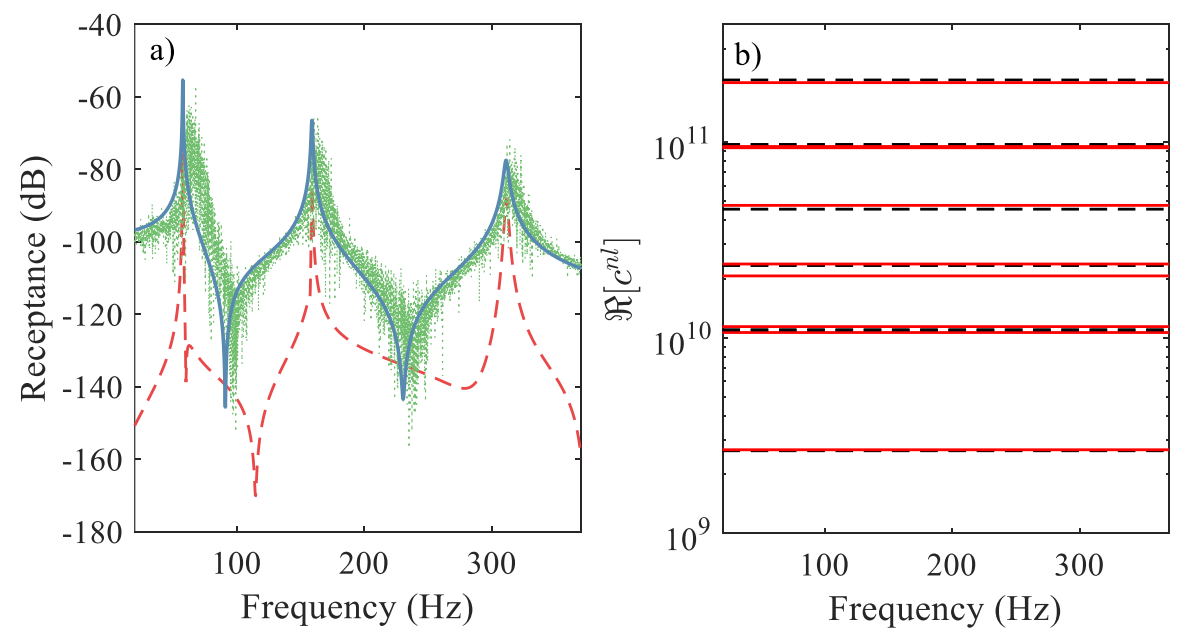

Fig. 1: Results of the identification. a) Blue line: driving point receptance of the identified underlying linear system; red dashed line: residue with the analytical linear receptance; green dotted line: nonlinear FRF distortion. b) Red lines: real part of the identified nonlinear coefficients; black dashed lines: true nonlinear coefficients.

\section{References}

[1] S. Marchesiello, L. Garibaldi, "A time domain approach for identifying nonlinear vibrating structures by subspace methods", Mechanical Systems and Signal Processing 22 (2008) 81-101.

[2] A.H. Nayfeh, P.F. Pai, "Linear and Nonlinear Structural Mechanics", Wiley-Interscience, New York, 2004.

[3] M. Amabili, M. Paidoussis, "Review of studies on geometrically nonlinear vibrations and dynamics of circular cylindrical shells and panels, with and without fluid-structure interaction", Applied Mechanics Reviews 56 (2003) 349381.

[4] G. Kerschen, K. Worden, A.F. Vakakis, J.C. Golinval, "Past, present and future of nonlinear system identification in structural dynamics", Mechanical Systems and Signal Processing 20 (2006) 505-592.

[5] P. Van Overschee, B. De Moor, "Subspace Identification for Linear Systems: Theory-Implementation-Applications", Kluwer Academic Publishers, Dordrecht, The Netherlands, 1996.

[6] S. Marchesiello, A. Fasana, L. Garibaldi, "Modal contributions and effects of spurious poles in nonlinear subspace identification", Mechanical Systems and Signal Processing 74 (2016) 111-132.

[7] J.P. Noël, G. Kerschen, "Frequency-domain subspace identification for nonlinear mechanical systems", Mechanical System and Signal Processing 40 (2013) 701-717.

[8] G. De Filippis, J.P. Noël, G. Kerschen, L. Soria, C. Stephan, "Model reduction and frequency residuals for a robust estimation of nonlinearities in subspace identification", Mechanical Systems and Signal Processing 93 (2017) 312-331.

[9] J.P. Noël, S. Marchesiello, G. Kerschen, "Subspace-based identification of a nonlinear spacecraft in the time and frequency domains”, Mechanical Systems and Signal Processing 43 (2014) 217-236. 